\title{
Como Implementar Cuidados Paliativos de Qualidade na Unidade de Terapia Intensiva*
}

\author{
How to Implement Quality in Palliative Care at Intensive Care Unit
}

\author{
Rubens C Costa Filho, FCCP, FCCS, MBA', João Luiz Ferreira Costa, MBA2, \\ Fernando Luiz B da R Gutierrez, PhD ${ }^{3}$, Ayla Farias de Mesquita, RN, MSc ${ }^{4}$
}

\section{RESUMO}

JUSTIFICATIVA E OBJETIVOS: Os cuidados paliativos têm como objetivo a prevenção e o alívio do sofrimento, melhorando a comunicação e o sinergismo com a terapêutica curativa. Esta filosofia de tratamento conjuga os valores dos pacientes e de seus familiares, facilitando as transferências entre os vários setores intra-hospitalares, que possam estar conduzindo um paciente com grave risco de vida.

CONTEÚDO: Apesar de ainda estar se desenvolvendo em todo o mundo, os cuidados paliativos estão progressivamente integrando-se aos cuidados curativos, inclusive nos ambientes de terapia intensiva. Entretanto, várias evidências têm demonstrado que esta abordagem médica ainda precisa melhorar, seja para os pacientes com sintomas de desconforto significativos ou dores físicas, nas unidades de terapia intensiva, ou na percepção dos familiares, que tam-

1. Chefe das Unidades de Terapias Intensivas do Hospital Pró-Cardíaco (HPC)

2. Chefe do Serviço de Nefrologia de Agudos e Depuração ExtraRenal (HPC); Coordenador de Rotinas das Terapias Intensivas do HPC; Professor Auxiliar da UNIRIO

3. Supervisor de Pesquisa da Terapia Intensiva do Hospital Pró-Cardíaco (HPC)

4. Enfermeira Chefe das Unidades de Terapias Intensivas do Hospital Pró-Cardíaco (HPC); Professora Assistente da UERJ

*Recebido do Centro de Terapia Intensiva Hospital Pró-Cardíaco, Rio de Janeiro, RJ

Apresentado em 10 de janeiro de 2008

Aceito para publicação em 03 de fevereiro de 2008

Endereço para correspondência:

Dr. Rubens C Costa Filho

Rua General Polidoro, 192 / 2 A - Botafogo

22280-003 Rio de Janeiro, RJ

Fone: (21) 2528-1433 - Fax (21) 2528-1414

E-mail: rubens1956@gmail.com; cti@procardiaco.com.br

(C)Associação de Medicina Intensiva Brasileira, 2008 bém é falha, sobretudo no que cerne às recomendações básicas como as intervenções diagnósticas, prognósticas e terapêuticas.

CONCLUSÕES: O desenvolvimento de pesquisa nesta área promoverá indicadores de desempenho, o que nos garantirá eficiência, qualidade operacional, e melhoria constante desses cuidados. Este artigo destaca a importância da medicina paliativa e propõe alternativas e planos para promoção de uma abordagem paliativa em tempo apropriado, no sentido de tornar a medicina geral mais próxima dos valores e dignidade humana.

Unitermos: alivio do sofrimento, Cuidados Paliativos, filosofia de tratamento, terminalidade e qualidade, valores do paciente.

\section{SUMMARY}

BACKGROUND AND OBJECTIVES: Palliative care has the objective of preventing and relieving suffering by improving communication and synergism with curative therapy. This philosophy of treatment combines the patients' values and their families and facilitates the movement in various hospital departments, which might occur with critically ill patients.

CONTENTS: Though still little developed in the world, palliative care is gradually integrating curative care in intensive care units. However, various pieces of evidence have been showing that there is still a necessity to improve, especially for patients with symptoms of discomfort and pain, in intensive care environments. The patients' families still have a poor understanding of the basic recommendations: diagnostic, prognostic and therapeutic interventions.

CONCLUSIONS: Development of research in this area can promote performance indicators trying to ensure efficiency, operational quality, and constant improvement of care. This article highlights the 
importance of palliative medicine and proposes alternatives to promoting an appropriate time approach, bringing general medicine closer to human values.

Key Words: End-of-Life and quality, Palliative Care, patients value, Philosophy of treatment, relieving suffering

\section{INTRODUÇÃO}

O verbo paliar, do latim palliare, pallium, significa em seu modo mais abrangente, proteger, cobrir com capa ${ }^{1}$. No entanto, paliar é mais usado em nosso meio, como aliviar provisoriamente, remediar, revestir de falsa aparência, dissimular, bem como adiar, protelar.

O cuidado paliativo (CP) ou paliativismo, é mais que um método, é uma filosofia do cuidar. O CP visa prevenir e aliviar o sofrimento humano em muitas de suas dimensões. O objetivo do CP é dar aos pacientes e seus entes queridos, a melhor qualidade possível de vida, a despeito do estágio de uma doença, ou a necessidade de outros tratamentos ${ }^{2}$. O CP complementa-se ao já tradicional cuidado curativo, incluindo objetivos de bem-estar e de qualidade de vida aos pacientes e seus familiares, ajudando-os nas tomadas de decisões, e promovendo oportunidades de crescimento e evolução pessoal. Esta filosofia de cuidar completa os tratamentos curativos da Medicina moderna, mas principalmente proporciona aos profissionais da área, dignidade e significado aos tratamentos escolhidos.

\section{SITUAÇÃO DOS CUIDADOS PALIATIVOS NO BRA- SIL E NO MUNDO}

Wright e col. ${ }^{3,4}$ realizaram uma pesquisa em 2006, relativa ao levantamento mundial dos $\mathrm{CP}$ no mundo. $\mathrm{O}$ relatório publicado foi destinado à Aliança Mundial de Cuidado Paliativo (World Wide Palliative Care Alliance) no sentido de melhorar suas ações e atividades organizadas.

Os autores utilizaram um sistema de 4 níveis de CP e mapearam todos os continentes do planeta. Em 35 países, a maioria no continente europeu, e em outros como Chile, Costa Rica, Argentina, Austrália e África do Sul, os CP se aproximaram e integraram aos serviços convencionais de saúde (nível 4). O Reino Unido destacou-se como a melhor relação de todo o continente europeu, com relação de 1 serviço de CP para cada 40.000 habitantes (1:40.000).
Na América Latina a melhor relação de serviços à população é da Costa Rica (1:166.000). O Brasil teve uma das relações mais pobres $(1: 13.315 .000)$ situando-se entre os 80 países com nível 3. Nosso país ainda não demonstrou possuir medidas de integração dos CP nos principais sistemas de saúde. $\mathrm{Na}$ classificação nível 1, onde não se obteve qualquer informação relevante sobre CP, foram encontrados 79 países. Este cenário pode ser explicado não somente pela presença de dificuldades locais, mas também pela má compreensão da filosofia que alimenta os CP (Figura 1).

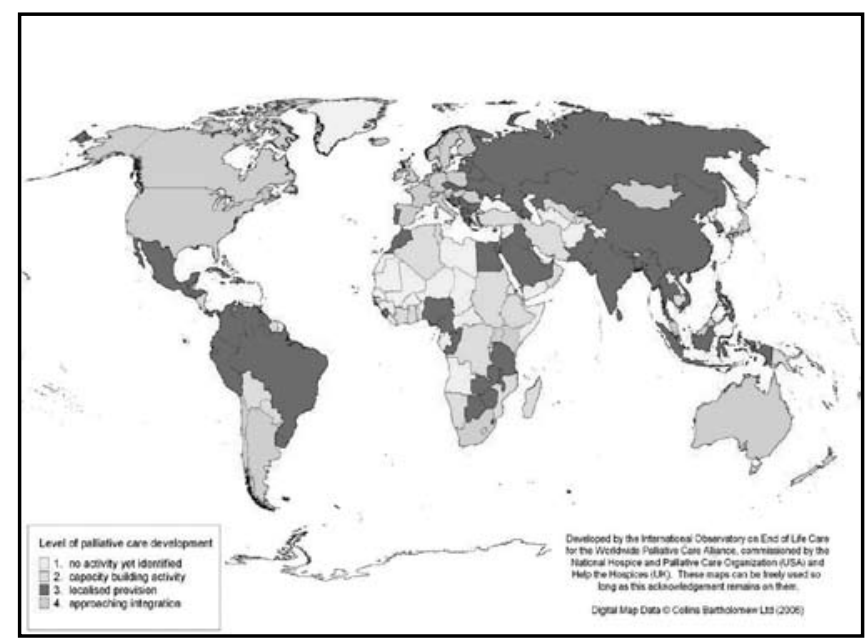

Figura 1 - O Brasil inclui-se entre os 80 Países no Mundo da Categoria de nível 3 (cinza escuro)- onde a Provisão dos Cuidados Paliativos e Hospice são Localizadas e está Completando a Integração com os Serviços de Saúde.

Um estudo recente identificou que os hospitais ingleses estão desenvolvendo um excelente trabalho no exercício dos cuidados paliativos ${ }^{5}$. Fármacos e tratamentos considerados não essenciais, por exemplo, foram interrompidas em $93 \%$ dos pacientes. O controle das dores e alívio foi obtido em $91 \%$ dos pacientes em seus últimos dias ou horas de vida. Todavia esses hospitais foram piores no que cerne a comunicar as más notícias. Somente $57 \%$ dos pacientes receberam notícias de que eles não viveriam muito tempo, e somente $45 \%$ reconheceram a natureza de suas condições. Neste cenário mais de $80 \%$ dos cuidadores estavam cientes do diagnóstico dos pacientes, indicando que a equipe poderia ter discutido ou conversado mais com seus pacientes, amigos e familiares.

Os CP, nos Estados Unidos, também vêm apresentando mudanças importantes com crescimento significativo nas últimas décadas (Figura 2). 


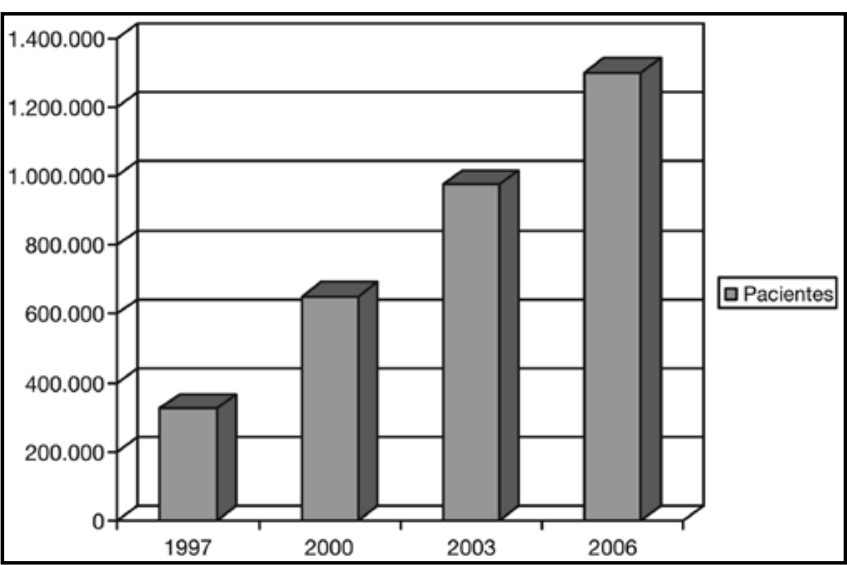

Figura 2 - Crescimento dos Cuidados Paliativos nos Estados Unidos.

Um recorde de 1,3 milhões de pessoas sendo atendidas nos hospices americanos. Um aumento de 100 mil pessoas sendo servidas por este sistema que envolve cuidados paliativos no período de 1 ano (2005 a 2006).

Adaptado do NHPCO http://www.nhpco.org/files/public/Statistics_Research/NHPCO_facts-and-figures_Nov2007.pdf e http:// www.nhpco.org/i4a/pages/index.cfm?pageid=3274

No brasil dispomos de poucas unidades dedicadas aos CP dentro de hospitais. O pioneiro foi iniciado em 1983, pela Dra. Miriam Martelete, do Departamento de Anestesiologia do Hospital das Clínicas da Universidade Federal do Rio Grande do Sul. Em 1986, na Santa Casa de Misericórdia de São Paulo, surgiu o Serviço de Dor e Cuidados Paliativos. Em 1989 surgiram o Centro de Estudos e Pesquisas Oncológicas (CEPON) em Florianópolis e o Grupo Especial de Suporte Terapêutico Oncológico (GESTO) no INCa no Rio de Janeiro. Até o momento não há mais do que 340 grupos no Brasil com unidades de cuidados paliativos dedicados e vinculados às instituições hospitalares ${ }^{6}$.

\section{A INCORPORAÇÃO E MODELOS DE CUIDADO PA- LIATIVO NA UTI}

O CP é uma abordagem terapêutica que envolve uma equipe multidisciplinar, incluindo várias especialidades médicas, enfermeiras, psicólogos, psiquiatras, nutricionistas, fisioterapeutas, fonoaudiólogos, assistentes sociais, farmacêuticos, conselheiros espirituais e sacerdotes. Todos esses profissionais são importantes, uma vez que a Medicina paliativa objetiva identificar e dirimir os problemas relacionados à internação, na esfera física, psicológica, espiritual ou social. Neste cenário, uma boa comunicação entre os envolvidos é fundamental.

Os CP devem integrar todos os setores de cuidados em saúde: emergências, unidades de terapia intensiva
(UTI), enfermarias, internações domiciliares - homecare e até Hospices, que no Brasil poderíamos chamar de instituições asilares nível 3. Quando existe uma equipe de $\mathrm{CP}$, ela colabora sinergicamente com outros profissionais de saúde, em cada um desses ambientes. Todos os pacientes, independente de suas idades, com diagnóstico de uma doença ameaçadora à vida, ou enfrentando uma condição debilitante são candidatos aos $\mathrm{CP}^{2}$. Desta forma, o paliativismo é apropriado principalmente para os pacientes que sofrem de câncer, insuficiência cardíaca grave progressiva, falência hepática e/ou renal, doenças neurodegenerativas como o Alzheimer, lesões medulares graves, doenças pulmonares crônicas e degenerativas, e inúmeras outras condições encontradas freqüentemente nas UTI, e também por vezes fora da terapia intensiva ${ }^{7}$.

Entretanto, surgem evidências na literatura sobre o controle inapropriado de sintomas, ao final de vida nas UTI. O controle da dor é um bom exemplo ${ }^{8-12}$. No estudo SUPPORT, com 9105 pacientes adultos em cinco hospitais de ensino americanos, a mortalidade global em seis meses foi de $47 \%{ }^{13}$. Observou-se que $50 \%$ dos pacientes que morriam apresentaram dores de grau moderado a grave nos últimos três dias de vida. Somente $41 \%$ destes pacientes conversaram com seus médicos sobre prognóstico e prescrições de "não reanimar" em caso de parada cardiorrespiratória. A maioria dos médicos (80\%) não compreendeu as preferências destes pacientes, pois quando os pacientes desejavam que as manobras de reanimação realmente fossem suspensas, elas não foram acatadas ou comunicadas à equipe de cuidados diretos.

Publicações demonstram que as necessidades dos familiares e pacientes internados nas UTI não estão sendo atendidas ${ }^{14,15}$. A comunicação entre médicos e familiares parece inadequada ${ }^{16,17}$ e muitos médicos estão despreparados para promover cuidados ideais aos pacientes terminais dentro das UTI ${ }^{18,19}$.

Famílias revelam que uma comunicação inconsistente recebida das equipes cuidadoras é uma das suas maiores preocupações. A falta de clareza e profundidade faz com que familiares reportem dissabores e ansiedade pela forma com que seus entes estão sendo tratados ${ }^{20-22}$.

A efetividade ou eficiência da Medicina Intensiva, tradicionalmente é demonstrada por dados de mortalidade ajustada por gravidade; por tempo de permanência; ou por desempenho operacional das equipes. No entanto, sob a perspectiva de qualidade do cuidado, a satisfação e o bem-estar são os aspectos importantes, 
especialmente dentro de ambientes de alta complexidade $^{23}$.

A Robert Wood Johnson Foundation Critical Care Endof-Life Peer Workgroup ${ }^{24}$ define seis domínios de qualidade nas UTI (Quadro 1).

Estas ações vão desde intervenções de enfermagem ligadas ao paliativismo até programas de treinamento integrado à rotina operacional da prática curativa. (www.promotingexcellence.org/i4a/pages/index. cfm?pageid=3276).

Assim, os CP podem ser ofertados dentro de diferentes modelos (Quadro 2).

Quadro 1 - Indicadores de Qualidade em Terapia Tntensiva (adaptado da referência 24 )

Modelo de decisões centrados na família e pacientes
Comunicação adequada entre as equipes, com os familiares e
os pacientes
Cuidado continuado e sustentado
Suporte emocional e prático aos pacientes e familiares
Suporte espiritual aos pacientes e familiares
Suporte emocional e organizacional as equipes de terapia in-
tensiva

Quadro 2 - Modelos de Cuidados Paliativos segundo o National Consensus Project (http://www.nationalconsensusproject.org/ Downloads.asp)

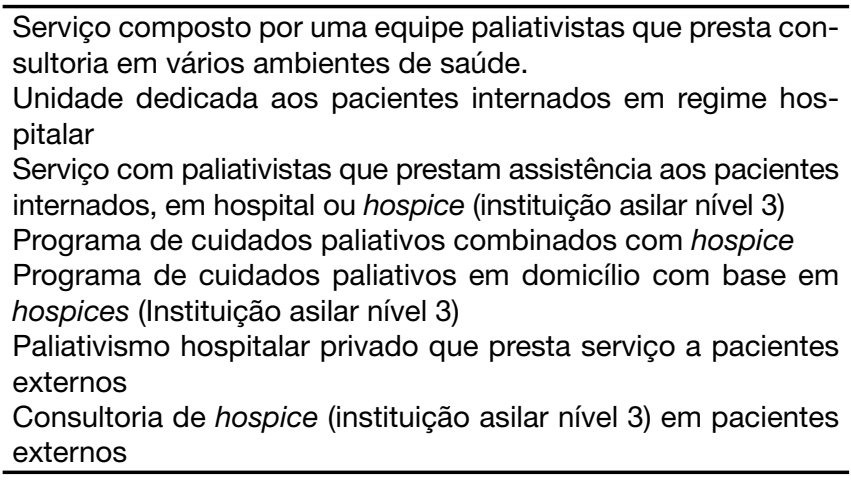

\section{EDUCAÇÃO E TREINAMENTO EM CUIDADOS PA- LIATIVOS NA UTI}

A dificuldade de comunicação entre os profissionais de saúde, os paciente e famíliares é sem dúvida um grande problema nas terapias intensivas. Um importante aspecto da Medicina é saber comunicar, informar relacionando-se com compaixão. A comunicação é o pilar da Medicina Paliativa. Comparada às medicações, uma habilidade em comunicação, possui eficácia paliativa; pode reduzir sintomas rapidamente sem efeitos colaterais. Assim, a comunicação de uma má notícia é um dos maiores desafios na prática diária dos profissionais das UTI. Má notícia é qualquer infor- mação que drástica e negativamente afeta a perspectiva de um indivíduo sobre seu futuro ${ }^{25-27}$.

As habilidades para a comunicação de uma má notícia foram entendidas por muitos anos como algo intuitivo, inato aos "bons médicos". Por décadas debateu-se se haveria técnicas para serem ensinadas ou aprendidas. $\mathrm{Na}$ verdade, a habilidade em apresentar uma má notícia, deve ser tecnicamente planejada e diversas estratégias podem ser aplicadas (www.breakingbadnews. co.uk). Neste portal estão descritas algumas, de como se deveria lidar com situações desagradáveis em nosso dia-a-dia, fruto das diferentes reações emocionais dos pacientes e seus familiares, após receberem uma má notícia. Tais reações se manifestam como a raiva, a culpa e acusação (contra à equipe de saúde ou aos mais próximos), o luto, os acordos de ocultação da verdade (colusão), sendo estas freqüentes nas UTI entre as famílias, e por último a negação.

Uma pesquisa indicou que muitos hospitalistas sentemse despreparados para indicar e prover CP e reconhecem a educação e treinamento como uma prioridade ${ }^{18}$.

\section{Alguns princípios para a comunicação de uma má notícia:}

- Escolher um momento em que o paciente e o médico estejam descansados e tenham um tempo adequado;

- Avaliar o estado emocional e psicológico do paciente;

- Preparar o paciente ou familiar dizendo ter um assunto difícil para discutir;

- Usar uma linguagem clara e simples;

- Expressar empatia pela dor do paciente;

- Ser humanitário tendo compaixão;

- Dar informação de forma gradual e programar outro encontro posterior;

- Ser realista evitando minimizar o problema, mas não tirar todas as esperanças;

- Verificar como o paciente e familiares sentem-se, depois de receber a notícia;

- Re-assegurar a continuidade do cuidado, não importando o que houver;

- Assegurar que o paciente tenha suporte emocional de outras pessoas.

O aperfeiçoamento de um diálogo estruturado deveria fazer parte do arsenal terapêutico das equipes, como maneira de prevenir dissabores ou atenuá-los ao máximo. Deveriam envolver vários profissionais neste contexto e treiná-los para lidar com essas situações que podem abalar o estado dos pacientes e da própria equipe de saúde. $O$ desenvolvimento de pesquisas e processos nesta área, pode promover indicares 
de desempenho que possam ser analisados, de modo a garantir eficiência, qualidade operacional e melhoria constante desses cuidados.

Finalmente, o crescimento da Medicina Paliativa, além das fronteiras hospitalares, com uma integração entre as equipes multidisciplinares à este contexto filosófico e humano, solidificaria e valorizaria mais ainda, nossas competências operacionais, à já estabelecida prática hospitalar.

\section{CONCLUSÃO}

A Medicina curativa nas UTI tem sido obstinada em ajudar a ganhar mais tempo de vida, mas ainda se omite em proporcionar qualidade de vida ao seu término. Não tem sido desenvolvida a atenção devida ao alívio do sofrimento dos pacientes terminais, pelo contrário, perde-se em discussões complexas, que oscilam entre eutanásias, distanásias e mistanásias, mesmo diante de um mundo globalizado, onde se convive com diversas culturas, religiões e moralidades.

A Medicina paliativa vai além destes rótulos; ela nos qualifica, desenvolve o aprendizado e ajuda a oferecer o que se tem de melhor, resgatando o amor.

A educação entre os profissionais de saúde, desde sua formação, com apropriada informação e treinamento torna-se fundamental para que o significado e a filosofia do exercício médico não se perca ${ }^{28}$. A promoção e a presença de uma abordagem paliativa aos pacientes elegíveis em tempo apropriado, certamente tornaria a Medicina geral mais próxima dos desejos e valores dignos dos homens.

\section{AGRADECIMENTOS}

À toda a equipe multidisciplinar de terapia intensiva do Hospital Pró-Cardíaco, verdadeiros abnegados, em especial à equipe de enfermagem da rotina, Marcela Calomeni, Sandra Regina Maciqueira Pereira e Theia Maria Forny Castellões, por sua incondicional e exemplar dedicação aos enfermos críticos e seus familiares. À todos os pacientes que lá deixaram tantas doenças para nosso aprendizado e formação intelectual, e àqueles que nos ensinaram com suas mortes, a enxergar mais com os olhos do coração.

\section{REFERÊNCIAS}

01. Houaiss A - Dicionário eletrônico da língua portuguesa versão 2.0. Editora Objetiva. http://www.dicionariohouaiss.com.br

02. Stoneberg JN, von Gunten CF et al - Assessment of palliative care nee- ds. Anesthesiol Clin, 2006;24:1-17.

03. Wright M, Wood J, Lynch T et al - Mapping levels of palliative care development: Associação Latino Americana de Cuidados Paliativos. Circular $\mathrm{N}^{\circ}$ 32 de 2007. http://cuidadospaliativos.org/archives/circularpor32.pdf.

04. Wright $\mathrm{M}$, Wood $\mathrm{J}$ - Mapping levels of palliative care development: a global view, 2006. http://www.nhpco.org/files/public/palliativecare/ World_map_report_final-0107.pdf

05. Kmietowicz Z - Dying patients are often not told of closeness of death - Nearly half of all patients dying in English hospitals are not aware they have only hours to live. BMJ, 2007;335:1176.

06. Figueiredo MTA. Reflexões sobre os cuidados paliativos no Brasil. Prática Hospitalar, 2006;47:36-40.

07. Reiriz AB, Scatola RP, Buffon VR et al - Cuidados paliativos, a terceira via entre eutanásia e distanásia: ortotanásia. Prática Hospitalar, 2006;48:77-82.

08. Lynn J, Teno JM, Phillips RS et al - Perceptions by family members of the dying experience of older and seriously ill patient. SUPPORT Investigators. Study to Understand Prognosis and Preferences for Outcomes and Risks of Treatments. Ann Intern Med, 1997;126:97-106.

09. Hawryluck LA, Harvey WR, Lemieux-Charles L et al - Consensus guidelines on analgesia and sedation in dying intensive care units patients. BMC Med Ethics, 2002;3:E13

10. Desbiens NA, Mueller-Rizner N - How well do surrogates assess the pain of seriously ill patients? Crit Care Med, 2000;28:1347-1352.

11. Desbiens NA, Wu AW, Broste SK et al - Pain and satisfaction with pain control in seriously ill hospitalized adults: findings from the SUPPORT research investigations. Crit Care Med, 1996;24:1953-1961.

12. Truog RD, Cist AF, Brackett SE et al - Recommendations for end-of-life care in the intensive care unit: The Ethics Committee of the Society of Critical Care Medicine. Crit Care Med, 2001;29:2332-2348.

13. A controlled trial to improve care for seriously ill hospitalized patients. The study to understand prognoses and preferences for outcomes and risks of treatments (SUPPORT). The SUPPORT Principal Investigators JAMA, 1995;274:1591-1598.

14. Azoulay E, Chevret S, Leleu G et al - Half of the families of intensive care unit patients experience inadequate communication with physicians. Crit Care Med , 2000;28:3044-3049.

15. Azoulay E, Pochard F, Chevret $\mathrm{S}$ et al - Impact of a family information leaflet on effectiveness of information provided to family members of intensive care unit patients: a multicenter, prospective, randomized, controlled trial. Am J Respir Crit Care Med, 2002;165:438-442.

16. Curtis JR, Patrick DL, Shannon SE et al - The family conference as a focus to improve communication about end-of-life care in the intensive care unit: opportunities for improvement. Crit Care Med, 2001;29:(Suppl1):N6-N33.

17. Abbott $\mathrm{KH}$, Sago JG, Breen $\mathrm{CM}$ et al - Families looking back: one year after discussion of withdrawal or withholding of life-sustaining support. Crit Care Med, 2001;29:197-201.

18. Plauth WH $3^{\text {rd }}$, Pantilat SZ, Wachter RM et al - Hospitalists' perceptions of their residency training needs: results of a national survey. Am J Med, 2001;111:247-254.

19. Tulsky JA, Chesney MA, Lo B et al - How do medical residents discuss resuscitation with patients? J Ger Intern Med, 1995;10:436-442.

20. Melltorp G, Nilstun T - The difference between withholding and withdrawing life-sustaining treatment. Intensive Care Med, 1997;23:1264-1267.

21. Carlet J, Thijs LG, Antonelli M et al - Challenges in end-of-life care in the ICU. Intensive Care Med, 2004;30:770-784.

22. Teno JM, Clarridge BR, Casey V et al - Family perspectives on end-of-life care at the last place of care. JAMA, 2004;291:88-93.

23. Stewart AL, Teno J, Patrick DL et al - The concept of quality of life of dying persons in the context of health care. J Pain Symptom Manage, 1999;17:93-108.

24. Clarke EB, Curtis JR, Luce JM et al - Quality indicators for end-of-life care in the intensive care unit. Crit Care Med, 2003;31:2255-2262.

25. Buckman R. - How to Break Bad News. Baltimore, Johns Hopkins Univ. Press, 1992.

26. Buckman R - Breaking bad news: why is it still so difficult? Br Med J, 1984;288:1597-1599.

27. Levinson W, Roter D - The effects of two continuing medical education programs on communication skills of practicing primary care physicians. J Gen Intern Med, 1993;8:318-324.

28. Rose N - Beyond medicalisation. Lancet, 2007;369:700-702. 